\title{
SAND99-0176C
}

\section{MOLECULAR DYNAMICS SIMULATIONS OF HEXADECANE/SILICALITE INTERFACES}

\author{
EDMUND B. WEBB III and GARY S. GREST
}

Corporate Research Science Laboratories, Exxon Research \& Engineering Company, Annandale, New Jersey 08801 and Sandia National Laboratories, Albuquerque, NM 87185-1411 [a]

\begin{abstract}
The interface between liquid hexadecane and the $(010)$ surface of silicalite was studied by molecular dynamics. The structure of molecules in the interfacial region is influenced by the presence of pore mouths on the silicalite surface. For this surface, whose pores are the entrances to straight channels, the concentration profile for partially absorbed molecules is peaked around 10 monomers inside the zeolite. No preference to enter or exit the zeolite based on absorption length is observed except for very small or very large absorption lengths. We also found no preferential conformation of the unabsorbed tails for partially absorbed molecules.
\end{abstract}

\section{INTRODUCTION}

Zeolites are inorganic crystalline solids with microporous networks, or channels, passing through them. These uniform channels make zeolites ideal materials for molecular separation and shape selective catalysis. The channels are created by the periodic repetition of eight, ten, or twelve member aluminosilicate rings (where the number refers to the number of cations in the ring). Channels formed in this manner have dimensions similar to the size of the molecules used in relevant chemical processes. This similarity places restrictions on which molecules can move into or out of the zeolite or which transition states can exist inside the zeolite. An example of this is found in the petrochemical industry where zeolites with channels formed by ten member rings $(10 \mathrm{MR})$ are used in the catalytic isomerization of linear alkanes. In this example, the size of the channels allows the formation of methyl branched molecules but restricts the formation of ethyl or larger branches. Shape selectivity results in more useful product distributions than are attained in the absence of a shape selective catalyst.

Because many applications involve the motion of molecules inside zeolite channels, a large body of research has been aimed at learning more about the behavior of molecules inside these structures [1-8]. Adsorption isotherms, diffusion constants, and activation energy for diffusion for a variety of molecule/zeolite combinations have been measured experimentally [1]. Other experiments [9-13] have focused on the catalytic reaction of ideal feed streams in the presence of a zeolite; the subsequent product distributions are used to characterize the molecule/zeolite system, often via comparison to a well known system. Studies such as these give insight to possible mechanisms for the relevant catalysis. However, there have been difficulties obtaining consistent results for diffusion from different experimental methods $[1,14]$. For some methods, there is still debate over whether the experiment measures properties reflecting molecular motion or some other physical process.

Computer modeling is an alternate method for studying molecular behavior inside zeolites. Molecular dynamics (MD) simulations generate a molecular trajectory and, as such, are very well suited to studying transport in zeolites. This method has been applied by various groups previously [5, 15-22]. Computational constraints have limited most of these studies to small molecules but diffusion for linear alkanes as large as C30 has been observed in various zeolites [23]. In all prior studies, intracrystalline diffusion alone was studied and most of the work was for infinite dilution or single molecule transport. There have only been a few attempts $[5,16,18,19,21]$ to study the effects of loading on transport due to the 
increased computational requirements. There has been no work addressing the equilibrium interface between a molecular liquid and a zeolite. Since nearly all zeolite applications involve molecules entering the zeolite channels to some degree and exiting again, we performed a study of interfacial dynamics in such a system. While some applications are gradient driven separations and are thus well addressed by nonequilibrium simulations, many catalysis operations involve extremely low fluxes. A feed stream may be in contact with a zeolite powder bed for minutes to hours and it is likely that near equilibrium interfaces are established throughout most of the reaction chamber. For this reason we present simulations of the interface between an alkane liquid and a zeolite.

The system chosen for this initial study is hexadecane in contact with silicalite, referred to in this work by its structure type code: MFI. Hexadecane was chosen because it is large enough to demonstrate properties of a flexible chain molecule at the temperature we studied. The zeolite MFI has a three dimensional pore structure composed of two distinct types of channels. Channels run in the [100] or [010] direction; the walls of channels running in the [100] direction have well pronounced oscillations so these channels are described as sinusoidal. Channels running in the [010] direction do not undulate and so are called straight. Intersections between straight and sinusoidal channels create pathways for diffusion in the third dimension. In the present work, we only study the (010) surface as this is the surface from which molecules can enter the zeolite most quickly.

\section{MODEL AND METHOD}

The alkane molecules were simulated using an united atom (UA) model in which $\mathrm{CH}_{n}$ groups are treated as single particles. The UA model used to describe alkane interactions is due to Siepmann et al. [24] with a torsional potential from Jorgensen et al. [25]. Interactions between hexadecane and the zeolite are represented by a Lennard-Jones (LJ) potential between $\mathrm{CH}_{n}$ monomers and the oxygens of the MFI lattice [26]. The cutoff used for all LJ interactions was $r_{c}=10 \AA$. The zeolite lattice was held rigid, a common simplification employed in prior simulations of diffusion in zeolites. The structure for MFI was obtained directly from the zeolite structure database contained in the Solids Builder module of the Insight II software package [27]. As such, the lattice used represents ideal stoichiometry and crystal structure.

Simulations presented here were done at $T=600 \mathrm{~K}$ and the hexadecane bulk density was the atmospheric pressure value for this temperature, $\rho=0.5621 \mathrm{gm} / \mathrm{cm}^{3}$. Temperature was maintained via a stochastic force thermostat with a time constant $\tau=1 \mathrm{ps}$. The equations of motion were integrated using the velocity Verlet algorithm and bond lengths were kept constant using the RATTLE algorithm [28]. A 5 fs time step was used in all simulations.

The initial state of the system was formed by placing a slab of hexadecane liquid, periodic in the $\mathrm{x}$ and $\mathrm{z}$ dimensions, between two (010) surfaces of MFI. The zeolite surfaces were connected by periodic boundary conditions but were sufficiently far apart to prevent molecules at one surface from sensing the other surface, thus forming two liquid/solid interfaces as seen in Fig. 1. Subsequent MD in the canonical ensemble allowed hexadecane molecules to enter the MFI, causing a drop in the bulk liquid density. The y-dimension of the system was scaled accordingly during equilibration to adjust the bulk density to its appropriate value. The system size was adjusted until a constant value of both bulk liquid density and molecular filling of the zeolite were obtained. The simulation time needed for equilibration was roughly 6 ns. Two interface systems were studied: one used a zeolite crystal with unit cell dimensions $2 \times 2 \times 2$ and the other was $4 \times 4 \times 4$. Figure 1 shows the smaller interface after equilibration; the small and large interface system dimensions were $40.0 \AA \times 94.7 \AA \times 40.1 \AA$ and $80.0 \AA \times 133.5 \AA \times 80.2 \AA$, respectively. A total of 160 and 760 hexadecane molecules were used in the small and large interface systems, respectively. 


\section{DISCLAIMER}

This report was prepared as an account of work sponsored by an agency of the United States Government. Neither the United States Government nor any agency thereof, nor any of their employees, make any warranty, express or implied, or assumes any legal liability or responsibility for the accuracy, completeness, or usefulness of any information, apparatus, product, or process disclosed, or represents that its use would not infringe privately owned rights. Reference herein to any specific commercial product, process, or service by trade name, trademark, manufacturer, or otherwise does not necessarily constitute or imply its endorsement, recommendation, or favoring by the United States Government or any agency thereof. The views and opinions of authors expressed herein do not necessarily state or reflect those of the United States Government or any agency thereof. 


\section{DISCLAIMER}

Portions of this document may be illegible in electronic image products. Images are produced from the best available original document. 


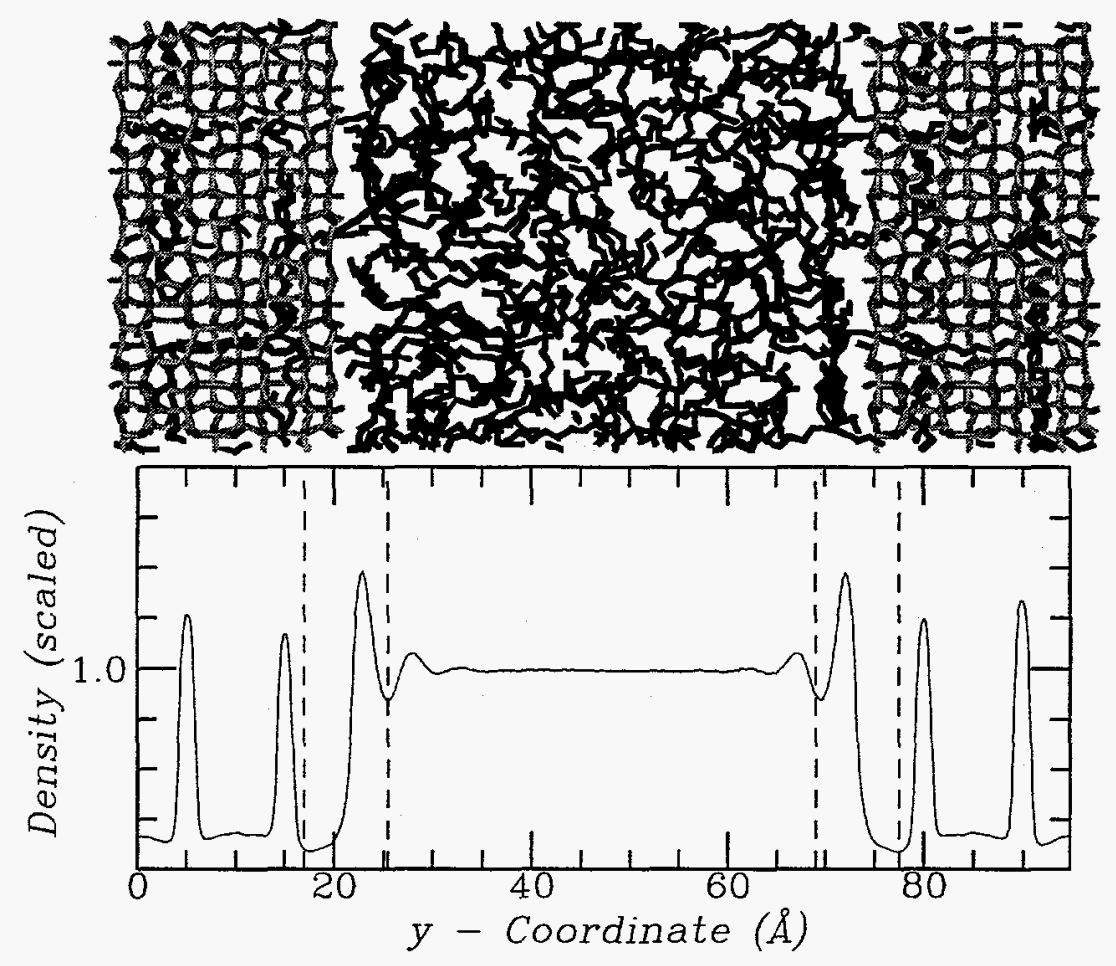

Figure 1: Snapshot of the small interface system at equilibrium; the liquid is sandwiched between the (010) crystal surfaces and the y-axis of the system lies horizontal in the figure. The figure was rendered by VMD [29]. The hexadecane density profile along the corresponding direction is shown in the lower panel.

The analysis results presented are from runs of 10 ns after equilibration.

A side view of the system is presented in the top panel of Fig. 1; in this perspective, the liquid is sandwiched between the MFI surfaces and hexadecane molecules can be seen throughout the channel structure of the crystal. Because of periodic boundary conditions, a molecule that enters the zeolite at one surface and diffuses all the way through to the other surface reenters the liquid. The horizontal direction in Fig. 1 corresponds to the $y$-axis of the simulation box. The hexadecane density profile along the $y$-direction is presented in the lower panel of Fig. 1. As seen in Fig. 1, the density is constant in the middle of the sample, demonstrating that the two interfaces are sufficiently far apart to prevent interaction between them. The density profile in Fig. 1 shows peaks near the two interfaces representing adsorbed liquid layers at the solid surfaces. This behavior is common for solid/liquid interfaces.

\section{RESULTS AND DISCUSSION}

The density profile in Fig. 1 allows us to delineate between different regions of the simulation cell. One already mentioned is the bulk region of the liquid. Besides ensuring that the interfaces do not interact with each other, we are not concerned with this region in the current study. Hexadecane density peaks inside the zeolite represent absorbed molecules. The uniform spacing between these peaks illustrates the periodic nature of the channel structure inside the crystal. At equilibrium, one also expects these peaks to have nearly uniform intensities which is the case in Fig. 1. The molecular filling after equilibration for both the small and large systems was approximately 4 molecules/unit cell. Studying the motion of absorbed molecules could elucidate aspects of diffusion or transport under high load conditions. However the time for molecules in the center of the zeolite to exit to the liquid was too slow for us to measure. That is, the diffusion constant at this high loading is below 


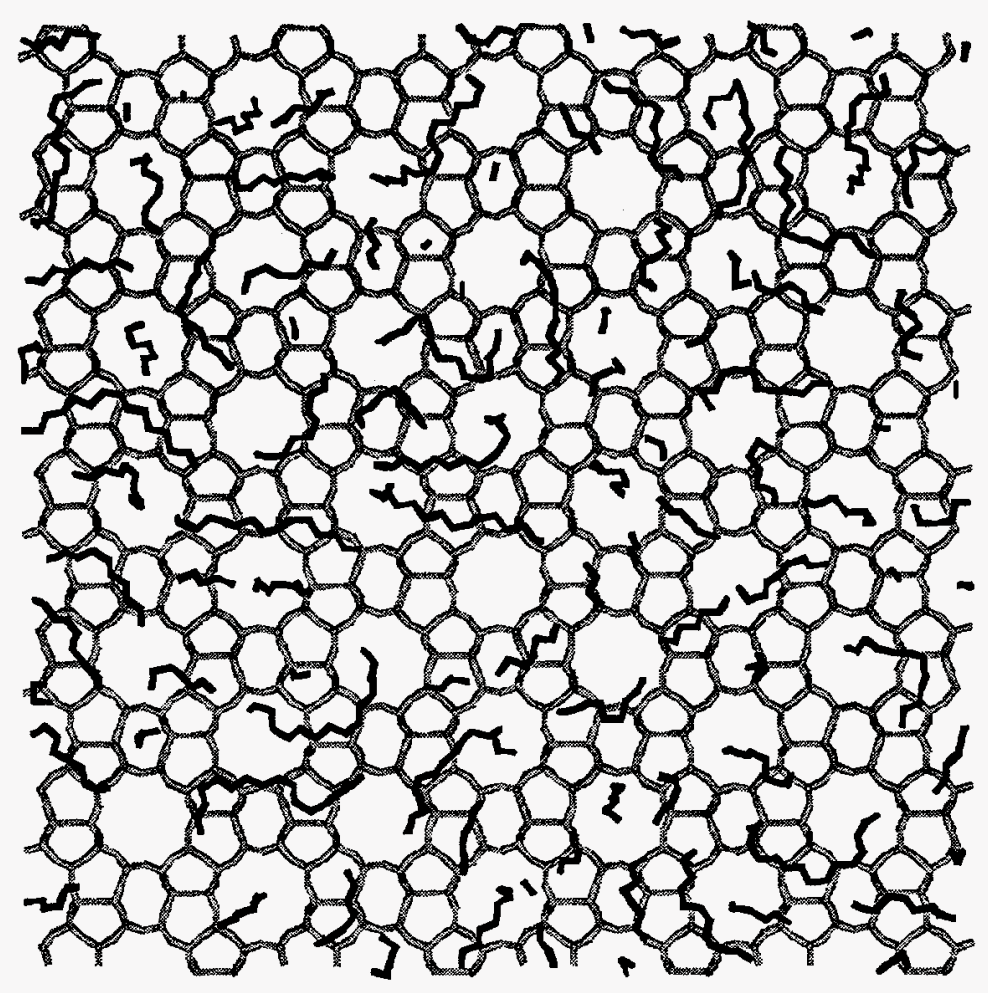

Figure 2: Surface adsorbed layer for the large system. Only molecular segments between one set of dashed lines on the density profile in Fig. 1 are shown.

the range that we can reliably measure. For this reason, we focus our present study on the interfacial region.

The surface adsorbed layers for both interfaces are indicated by the dashed lines in Fig. 1. These are drawn through the density minimums on either side of each peak, although their exact position does not significantly influence the results we are presenting. Figure 2 shows the hexadecane molecular segments comprising the surface adsorbed layer for one interface. The snapshot is taken looking down on the zeolite surface which is also drawn for clarity. From this illustration, it can be seen that there are three general descriptions of molecules with segments in this region. Some lie completely on the zeolite surface, preferentially avoiding the void spaces at pore openings. This maximizes their interaction with the zeolite surface oxygens. For other molecules, only part of their length is lying on the zeolite surface with the remainder either sticking into the zeolite, the liquid, or both. The third group of molecules do not lie on the zeolite surface at all and are straddling the surface adsorbed layer with one end inside the zeolite and the other sticking further into the liquid. Such an adsorption layer is quite different than that seen in simulations of hexadecane on fcc (001) gold where the linear chains are more ordered and planar [30]. Clearly the pore structure disrupts the ability of the molecules to order.

In the current study, molecules with some segment inside the zeolite are referred to as partially absorbed. The amount of absorption can be quantified according to the number of united atoms, or monomers, that are inside the zeolite. When this number equals sixteen for a given molecule, we considered it to be completely absorbed. While we will present additional results for these systems in the future, here we focus on the nature and eventual fate of these partially absorbed molecules. While the data presented is from the large interface system, for the features we present, there is no difference between the two systems. 

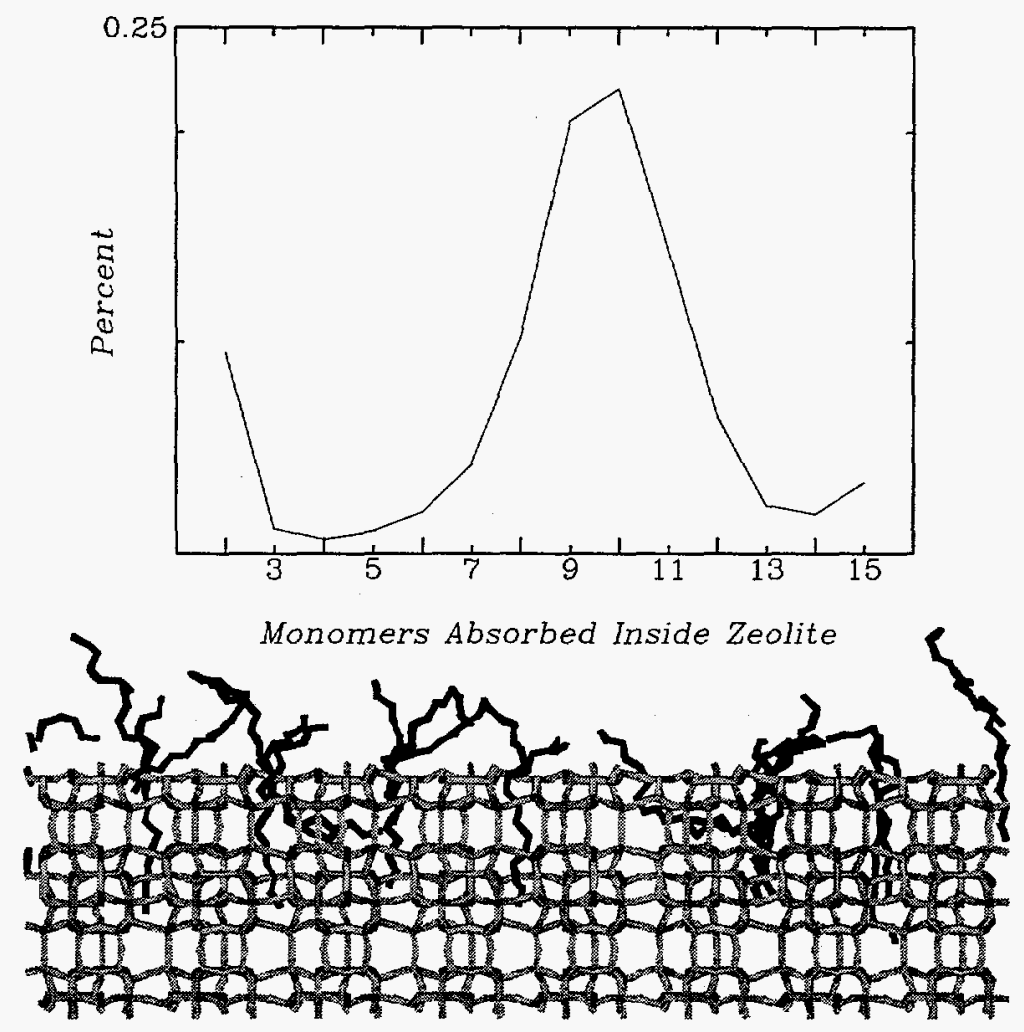

Figure 3: The top panel shows the concentration profile of partially absorbed molecules versus absorption length. The lower panel is a side view of the partially absorbed molecules at one surface. Because this is an orthographic projection, molecules separated along the dimension into the page appear to lie on top of one another.

The top panel in Fig. 3 shows the time average concentration profile of partially absorbed molecules. The lower panel of Fig. 3 is a snapshot of one interface from the side illustrating the partially absorbed molecules. The snapshot is an orthographic projection which makes many molecules appear to have absorbed in each channel opening; this is only a result of the projection, as is evidenced by the top view in Fig. 2. The profile in Fig. 3 is clearly not uniform across absorption lengths. This shape is largely dictated by the structure of the zeolite and the surface termination. As stated previously, the $\mathrm{CH}_{3}$ groups on alkanes strongly influence the conformation of molecules in MFI. While this was observed in simulations in the dilute limit $[16,26]$, one expects that there will still be some influence at this loading level. The $\mathrm{CH}_{3}$ groups preferentially avoid the void spaces at intersections between the straight and sinusoidal channels of MFI. In the range of $3-5$ monomers absorbed, an end group resides in a void space roughly $4-6 \AA$ below the surface; since this is preferentially avoided, there is a minimum in the distribution at these absorption lengths. The same is true for absorption lengths in the range $13-15$ because the next intersection lies roughly $14-16 \AA$ below the surface. However, as can be seen in Fig. 3b, molecules with this many absorbed monomers may have turned the corner at the first intersection below the surface. In this fashion, they are partially absorbed in a straight channel and partially in a sinusoidal channel. This disrupts the spacing arguments above so that the minimum in the profile at the high absorption lengths is not as low as the minimum around $3-5$ monomers absorbed. The position of the maximum in the profile is roughly halfway between the minimums and this is sensible since this is the length at which molecules can minimize the effect of the intersections, or void regions. We are currently gathering data on interfaces with the other 

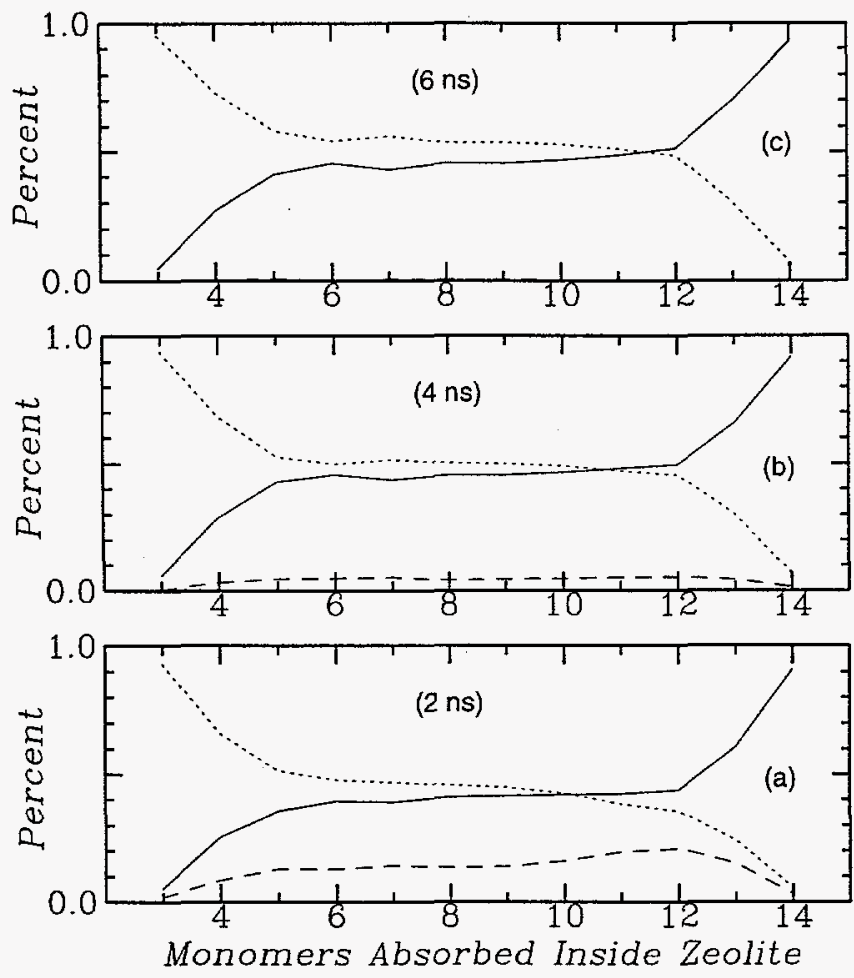

Figure 4: Concentration profiles versus absorption length for molecules that enter the zeolite completely(solid curve), exit completely (dotted), or do neither (dashed) in the time duration indicated. Analysis is for time windows of durations (a) 2, (b) 4, and (c) 6 ns.

low index terminations of MFI and the initial results indicate this concentration profile changes significantly.

To study the eventual fate of these partially absorbed molecules, we followed their motion until the point where they had either completely entered or exited the zeolite. Figure 4a shows three distributions for molecules across the range of absorption lengths. The solid curve shows the percentage of molecules at a given absorption length that eventually enter the zeolite in a preset time window of $2 \mathrm{~ns}$. The dotted line shows the percentage of molecules that eventually exit and the dashed line shows the percentage that do neither in the time allowed (i.e. they do not make up their mind). This is not to imply that the molecules which remain partially absorbed stay at the same absorption length throughout the $2 \mathrm{~ns}$; they do not. It simply means they do not either fully enter or exit the zeolite. Figures $4 \mathrm{~b}$ and $\mathrm{c}$ show the same result as the time window is increased to 4 and $6 \mathrm{~ns}$, respectively. Most importantly, it can be seen that, when the time window is increased to $6 \mathrm{~ns}$, the remainder profile goes essentially to zero. This provides us a measure of the time for partially absorbed chains to either exit or enter the zeolite at this temperature and density.

From Fig. 4c we see that there is no preference for molecules to either enter or exit the zeolite, except for very small and large absorption lengths. From an absorption length of 5 to 12 , there is roughly an equal probability of entering or exiting the zeolite. Since this system is in equilibrium and molecular motion is that of a random-walk nature, this is not greatly surprising. However, it is not strictly true that the chance for entering or exiting is equal. As the absorption length increases from 5 to 12 there is a gradual steady increase in the probability of entering and a corresponding decrease in the probability of exiting. The curves cross between absorption lengths 11 and 12. This is a subtle effect and we feel it is also related to the channel structure near the surface. Similar analyses on the 


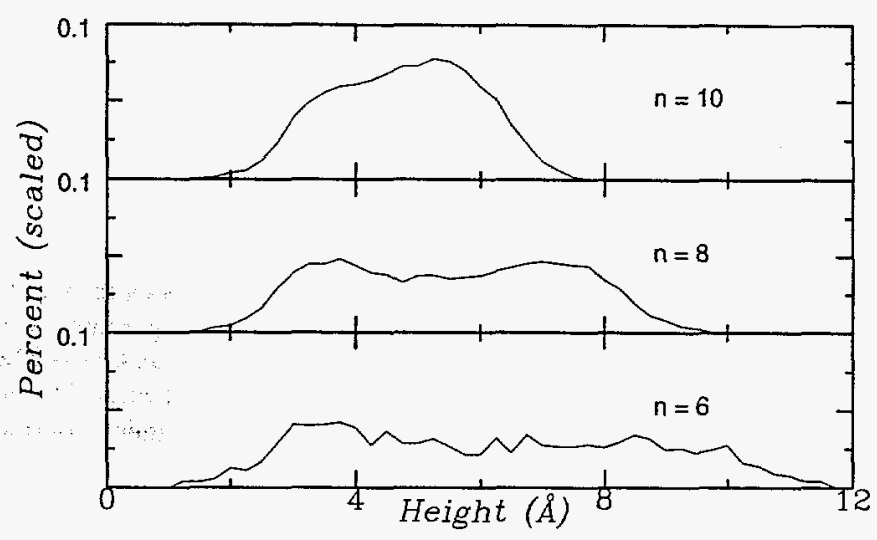

Figure 5: Height distributions for the unabsorbed ends of partially absorbed molecules. Data are shown for different amounts of absorption, $n$, where $n=6,8$, and10 monomers absorbed.

other crystallographic surfaces of MFI will assist in understanding this better. The most pronounced effect is that there is little preference to enter or exit at most absorption lengths except for when the molecule is either absorbed very little or nearly completely.

Partially absorbed molecules have, of course, some segment which is not inside the zeolite, or unabsorbed. To characterize partially absorbed molecules further we charted the conformation of their unabsorbed tails. This analysis was done in a fairly straightforward fashion: we measured the height of the unabsorbed tail end above the surface. If the unabsorbed tail of a molecule is lying on the zeolite surface, it's end height will be small. On the other hand, if the partially absorbed molecule is sticking into the zeolite pore with its unabsorbed end sticking into the liquid, the end height will be large. The range of heights narrows with greater absorption lengths as can be seen in Fig. 5. These curves are the unabsorbed tail end height distributions for absorption lengths of 6,8 , and 10 . Zero height is defined as the $y$-coordinate of the surface oxygens for MFI. The range covered by the profiles decreases as expected at larger absorption lengths. It is also obvious from these curves that there is no preference for certain conformations of the unabsorbed tail. The distributions are fairly uniform from a height representing tails which are lying on the surface to a height corresponding with the unabsorbed tail sticking straight out into the liquid. Although the width of the distribution narrows at higher absorption lengths, the uniformity remains.

Concerning the dynamic behavior of partially absorbed molecules, we investigated if a preferential conformation for entering (or exiting) the zeolite existed. One way of assessing this was to recalculate the profiles of Fig. 5 but only for those molecules that, in a time frame of $6 \mathrm{~ns}$, entered the zeolite. By comparing this distribution to the original distribution, we were able to determine if a particular conformation favored entering the zeolite. While the data is not presented, our results show that no such conformation existed: the profiles for chains which eventually entered the zeolite had nearly the exact shape of the original distributions for all absorption lengths. The intensity of these profiles was half of the original profiles representing the roughly equal likelihood of entering or exiting that was observed in Fig. 4c.

\section{CONCLUSIONS}

We have demonstrated the feasibility of studying the fairly complex system of an interface between an alkane liquid and a microporous solid. Our studies so far have focused on the interfacial region, specifically those molecules which are partially absorbed into the zeolite. The concentration profile for such molecules is not uniform across absorption lengths and this is at least partially a function of the zeolite channel structure and surface termination. 
We have observed no preference to enter or exit the zeolite based on absorption length except at very small and large absorption lengths. Furthermore, we see no preferential conformation of the unabsorbed tails of partially absorbed molecules. This is true for the static average as well as when this is only measured over those molecules that eventually enter the zeolite. In subsequent work we will address the effects of zeolite surface termination and state point more thoroughly.

\section{REFERENCES}

Sandia is a multiprogram laboratory operated by Sandia Corporation, a Lockheed Martin Company, for the United States Department of Energy under contract DE-AC04-94AL85000.

a. Present address for both authors.

1. J. Kärger and D. Ruthven, Diffusion in Zeolites and Other Microporous Solids (Wiley, New York, 1991).

2. W. J. M. van Well et al., J. Phys. Chem. B 102, 3952 (1998).

3. S. C. Reyes et al., J. Phys. Chem. B 101, 614 (1997).

4. M. A. Jama, M. P. F. Delmas, and D. M. Ruthven, Zeolites 18, 200 (1997).

5. R. Q. Snurr and J. Kärger, J. Phys. Chem. B 101, 6469 (1997).

6. B. G. Silbernagel, A. R. Garcia, and J. M. Newsam, Coll. Surf. A 72, 71 (1993).

7. W. Niessen and H. G. Karge, Appl. Surf. Sci. Catal. 60, 213 (1991).

8. P. Voogd and H. V. Bekkum, Appl. Catal. 59, 311 (1990).

9. P. Meriaudeau et al., J. Catal. 169, 55 (1997).

10. W. Souverijns et al., Stud. Surf. Sci. Catal. 105, 1285 (1997).

11. E. J. P. Feijen, J. A. Martens, and P. A. Jacobs, Stud. Surf. Sci. Catal. 101, 721 (1996).

12. J. A. Martens et al., Angew. Chem. Int. Ed. Engl. 34, 2528 (1995).

13. J. Weitkamp and S. Ernst, Catalysis Today 19, 107 (1994).

14. T. A. Nijhuis et al., Chem. Eng. Sci. 52, 3401 (1997).

15. A. Bouyermaouen and A. Bellemans, J. Chem. Phys. 108, 2170 (1998).

16. R. C. Runnebaum and E. J. Maginn, J. Phys. Chem. B 101, 6394 (1997).

17. F. Jousse, L. Leherte, and D. P. Vercauteren, J. Mol. Catal. A: Chem. 119, 165 (1997).

18. D. Keffer, A. V. McCormick, and A. T. Davis, Mol. Phys. 87, 367 (1996).

19. G. Schrimpf, B. Tavitian, and D. Espinat, Revue de L'Institut Francais du Petrole 50, 105 (1996).

20. D. Dumont and D. Bougeard, Zeolites 15, 650 (1995).

21. R. L. June, A. T. Bell, and D. N. Theodorou, J. Phys. Chem. 96, 1051 (1992).

22. C. R. A. Catlow et al., J. Chem. Soc. Faraday Trans. 87, 1947 (1991).

23. E. B. Webb III and G. S. Grest, J. Phys. Chem. B, submitted (1998).

24. J. I. Siepmann, S. Karaborni, and B. Smit, Nature 365, 330 (1993); B. Smit, S. Karaborni, and J. I. Siepmann, J. Chem. Phys. 102, 2126 (1995).

25. W. L. Jorgensen, J. D. Madura, and C. J. Swenson, J. Am. Chem. Soc. 106, 6638 (1984).

26. E. B. Webb III and G. S. Grest, Catalysis Letters, in print.

27. Molecular Simulations Inc., San Diego, CA.

28. M. P. Allen and D. J. Tildesley, Computer Simulation of Liquids (Clarendon, Oxford, 1987).

29. W. Humphrey, A. Dalke, and K. Schulten, J. Molec. Graphics 14, 33 (1996).

30. T. K. Xia, J. Ouyang, M. W. Ribarsky, and U. Landman, Phys. Rev. Lett. 69, 1967 (1992). 